Goldschmidt 2021 Abstract

https://doi.org/10.7185/gold2021.7311

\section{Maximizing precision and accuracy of TIMS Nd isotope measurements for small sample sizes}

\author{
LUKÁŠ ACKERMAN ${ }^{1}$, JAN REJŠEK ${ }^{1}$, JENNY ROBERTS ${ }^{2}$, \\ LAURA BRACCIALI ${ }^{2}$, HAUKE VOLLSTAEDT ${ }^{2}$, FILIP \\ SCHEINER $^{1}$ AND KATARÍNA HOLCOVÁ ${ }^{3}$ \\ ${ }^{1}$ The Czech Academy of Sciences \\ ${ }^{2}$ Thermo Fisher Scientific \\ ${ }^{3}$ Faculty of Science, Charles University \\ Presenting Author: ackerman@gli.cas.cz
}

Maximizing precision and accuracy of TIMS Nd isotope measurements for small sample sizes

Lukáš Ackerman ${ }^{1}$, Jan Rejšek ${ }^{1}$, Jenny Roberts ${ }^{2}$, Laura Bracciali $^{2}$, Hauke Vollstaedt ${ }^{2}$, Filip Scheiner ${ }^{1,3}$, Katarína Holcová $^{3}$

${ }^{1}$ Institute of Geology of the Czech Academy of Sciences, Czech Republic

${ }^{2}$ Thermo Fisher Scientific, Germany

${ }^{3}$ Faculty of Science, Charles University, Czech Republic

The neodymium $(\mathrm{Nd})$ isotope $\left({ }^{143} \mathrm{Nd} /{ }^{144} \mathrm{Nd}\right)$ composition of foraminifera is widely used as a tracer of oceanic mixing on glacial inter-glacial timescales, and of continental weathering on multi-million year timescales.

The availability of foraminifera and its different species in sampled sedimentary materials can limit the precision of $\mathrm{Nd}$ isotope measurements accelerating the increasing need to measure high-precision $\mathrm{Nd}$ isotope data for small sample sizes $(<1 \mathrm{ng} \mathrm{Nd})$. Recent advances in amplifier technology have enabled precise determination of small signals, improving the precision of small sample isotopic measurements $[1,2,3,4]$. For Nd metal determined by Thermal Ionization Mass Spectrometry (TIMS), precision better than 100 ppm (2 RSD) have been achieved for a $100 \mathrm{pg} \mathrm{Nd}$ loads of standard reference materials $[1,2]$ and recent Mediterranean foraminifera with an estimated $\mathrm{Nd}$ load $<1 \mathrm{ng}$. For these measurements, the precision is mainly limited by the counting statistics and hence the $\mathrm{Nd}$ ion yield (typically 2-3\%). In comparison, analyzing $\mathrm{Nd}$ isotopes in the oxide form $\left(\mathrm{NdO}^{+}\right)$by loading the samples with a tantalum oxide phosphoric acid slurry has been shown to yield much higher yields $(>10 \%$; [5]).

In this study we combine the $\mathrm{NdO}^{+}$method with the low noise Thermo Scientific $10^{13} \Omega$ amplifiers on a Triton series TIMS to obtain best accuracy and precision. The results demonstrate that high-precision $\mathrm{Nd}$ isotope data can be obtained for $\mathrm{Nd}$ loadings of $<1 \mathrm{ng}$, opening up the possibility to measure increasingly smaller foraminifera samples.

[1] Koornneef et al., Anal. Chem. Acta, 2014, doi: 10.1016/j.aca.2014.02.007

[2] Vollstaedt et al., Thermo Scientific App. Note 30493, 2018

[3] Adams et al., Nature Comm., 2021 doi: 10.1038/s41467021-21416-9

[4] Reinhard et al., Rapid Commun. Mass Spectrom., 2020, doi: $10.1002 / \mathrm{rcm} .9032$
[5] Harvey and Baxter, Chem. Geol., 2009, doi: 10.1016/j.chemgeo.2008.10.024

This research was supported by the Czech Science Foundation project 20-05872S. 